\title{
LA INTERSUBJETIVIDAD EN LA SEMÁNTICA DE ALGUNOS MARCADORES DE EVIDENCIA
}

(Intersubjectivity in the semantics of some evidentiality markers)

\author{
Enrique Huelva UnTERNBÄUMEN \\ (Universidade de Brasília)
}

\begin{abstract}
Resumo: Este estudo analisa a semântica dos seguintes marcadores de evidência da língua espanhola: claro, desde luego, naturalmente $e$ por supuesto. A partir da análise de enunciados reais, demonstra-se que a estrutura semântica de tais marcadores compõe-se de uma série de instruções para especificar o sentido do enunciado, em que são utilizados, com relação a um elemento central da base de conceituação ('Ground'): a intersubjetividade. Sustenta-se, ainda, que os efeitos de argumentativos que esses elementos produzem na conexão entre enunciados (os chamados efeitos argumentativos) derivam da mencionada função especificadora.
\end{abstract}

Palavras-chave: marcadores de evidência; ancoragem (grounding); intersubjetividade; argumentatividade.

\begin{abstract}
In this paper, we analyze the semantics of the following evidentiality markers in Spanish: claro, desde luego, naturalmente and por supuesto. Based on the analysis of real utterances, we show that the semantic structure of these markers consists of a series of instructions to specify the meaning of the utterance in which they occur, with respect to an essential element of the conceptualization base (the ground): intersubjectivity. We further argue that the meaning effects that these elements provoke in the connection between utterances (the so-called argumentative effects) are derived from this specifying function.
\end{abstract}

Key-words: evidence markers; grounding; intersubjectivity; argumentativity.

\section{INTRODUCCIÓN}

No es, de forma alguna, exagerado afirmar que el concepto de la intersubjetividad es uno de los que más intensamente ha marcado el desarrollo de la filosofía y de las ciencias humanas y hermenéuticas en las últimas décadas. En las ciencias lingüísticas su acogida fue también bastante temprana, concomitante prácticamente con el surgimiento de las disciplinas dedicadas al estudio de la conversación, del diálogo o del discurso. 
En el seno de estas disciplinas, la intersubjetividad designa, en general, el conocimiento compartido por los interactantes (shared knowledge), y esto en un doble sentido (cf. v.gr. Schegloff 2006): (i) el conocimiento compartido al que, por lo menos parcialmente, se puede llegar mediante los procesos de negociación de sentido que se llevan a cabo en la comunicación o (ii) como aquel conocimiento contextual y cotextual que se presupone como dado y compartido, en un momento determinado del proceso comunicativo, y que constituye el punto de partida y el horizonte interpretativo mutuo para la prosecución de la actividad comunicativa. Común a ambas perspectivas es la idea de la intersubjetividad como producto o efecto de la comunicación: producto al que se ha llegado gracias a procesos comunicativos pasados o al que se pretende llegar a través del que está actualmente en marcha.

Más recientemente, algunos autores han empezado a reconocer que la intersubjetividad posee además una relevancia gramatical, esto es, que representa una categoría conceptual codificable y codificada por elementos o construcciones gramaticales (Verhagen 2005; 2008; Huelva-Unternbäumen en prensa). ${ }^{1}$ Las consecuencias teóricas y metodológicas de esta constatación son enormes, así como - me atrevería incluso a decir - los desafíos que conlleva para la investigación lingüística.

Una de ellas, tal vez la más importante, repercute directamente en la propia definición del concepto de la intersubjetividad. Pues, como concepto codificado gramaticalmente, la intersubjetividad no puede ser concebida como el producto o efecto de un proceso comunicativo actual. No solemos comunicarnos para ponernos de acuerdo sobre el contenido semántico de los elementos o las construcciones gramaticales de nuestra lengua, a no ser que seamos lingüistas y lo hagamos ex oficio.

En el presente trabajo pretendemos asumir ese desafío y proponer una definición de la intersubjetividad como categoría codificada por la gramática. En ello reside el primer objetivo de esta investigación. Para alcanzarlo, será necesario, en primer lugar, asentar los fundamentos conceptuales generales que nos permitan concebir la intersubjetividad como una categoría central

1. Valgan aquí como ejemplos representativos los siguientes trabajos: Verhagen (2005) analiza la codificación de la intersubjetividad por partículas de negación, oraciones subordinadas sustantivas, y conjunciones (conectores) causales y consecutivas de diversas lenguas; Sanders / Sweetser (2009) muestran cómo la intersubjetividad es imprescindible para caracterizar la semántica (y la pragmática) de algunos conectores causales en holandés; Huelva-Unternbäumen (en prensa) constata que la intersubjetividad es un aspecto constitutivo de la semántica de la preposición ante en sus diferentes usos causales. 
de nuestro sistema lingüístico y, como tal, como un prerrequisito para la comunicación, en vez de como un producto inmediato de la misma. Esta fundamentación general la llevaremos a cabo recurriendo, especialmente, a la noción fenomenológica de la intersubjetividad, tal y como la propuso, originalmente, Edmund Husserl.

Hecho esto, estaremos en condiciones de plantearnos, en segundo lugar, la pregunta más concreta a cerca de cómo la gramática codifica esta categoría. Responder esta pregunta supone, por una parte, caracterizar la intersubjetividad en comparación con otras categorías codificadas gramaticalmente. Como veremos, las características que constituyen su especificidad como categoría codificada por la gramática nos dan margen para considerar que se trata de un tipo particular (y esencial) de lo que en la Lingüística Cognitiva se denomina Grounding (Langacker 1987: 126129; 2008: 259-309). Por otra parte, la respuesta a la pregunta planteada significa, obviamente, también identificar elementos gramaticales cuya función resida justamente en codificar esta categoría.

El segundo objetivo que nos proponemos es demostrar que los marcadores de evidencia (claro, desde luego, naturalmente, por supuesto, etc.) son elementos que desempeñan esta función. Tradicionalmente, los marcadores de este tipo han sido estudiados principalmente con relación a su contribución a la organización de la estructura argumentativa de un texto oral o escrito (cf. v. gr. Fuentes 1993a; 1993b; Martín / Portolés 1999: 41484158). En el presente trabajo defenderemos la tesis de que los marcadores de evidencia son también indicadores de intersubjetividad. Intentaremos demostrar, además, que su función como indicadores de intersubjetividad es, hasta cierto punto, prioritaria frente a las que ejercen en el plano de la argumentatividad. Dicho de un modo más concreto: el significado que aporta un determinado marcador de evidencia a la estructura argumentativa reposa sobre una configuración de intersubjetividad que el hablante y el oyente crean a partir de las instrucciones conceptuales codificadas por el propio marcador. Veamos, para ilustrar y concretar un poco más esta tesis, algunos ejemplos con el marcador naturalmente:

(1) En TVE se optó, entonces, por otra fórmula: una serie de periodistas, en su mayoría ajenos a la plantilla de RTVE, entrevistarían por separado a los candidatos. El programa estrella fue, naturalmente, el que tuvo como protagonista a Felipe González. ${ }^{2}$

2. Los enunciados analizados en el presente trabajo pertenecen al CREA (www.rae.es). 
Naturalmente actúa como un reforzador de la aserción del fragmento en que se utiliza (Martín / Portolés 1999: 4154-4155). La posibilidad de desempeñar esta función se debe a que el marcador convoca un determinado conocimiento contextual, lo presenta como intersubjetivamente (o incluso, en algunos casos, como universalmente) válido y establece una conexión de consecuencia 'natural' entre dicho conocimiento contextual y la aserción del fragmento en que aparece. En el ejemplo que nos ocupa, el hablante refuerza la validez de la aserción el programa estrella fue el que tuvo como protagonista a Felipe González conectándola con el hecho de que Felipe González es, 'como se sabe', el candidato más popular, que levanta mayores expectativas, etc.

Son varios los efectos argumentativos y discursivos que reposan sobre la validez intersubjetiva activada mediante el uso de naturalmente. Uno de los más habituales se manifiesta en la respuesta a preguntas absolutas:

(2) Cuando me introducen al calabozo-ventana, me preguntan:

- ¿Prefiere usted seguir en esta celda solo, o que lo reunamos con sus compañeros?

- Deseo estar con mis compañeros, naturalmente.

- Bueno, entonces vamos para allá -me responde el jefe de los guardias.

Estar con los compañeros se presenta como algo universalmente preferible, como la opción que pertenece al sentido común, privándole a la alternativa, consecuentemente, de cualquier posibilidad de ser llevada en cuenta como algo realizable bajo las circunstancias dadas.

El marcador naturalmente se emplea a menudo también con carácter profiláctico como bloqueador o inhibidor de una posible objeción del interlocutor (cf. v.gr. Martín / Portolés 1999: 4154-4155).

(3) Potencialmente, todo ser es infinito y eterno o, en otros términos, todo ser es, en esencia, el propio Absoluto. Naturalmente, eso no significa que haya tantos absolutos como seres, lo que sería absurdo.

En estos casos, el hablante se anticipa a una posible reacción de su interlocutor y confirma de ante mano lo que, según su apreciación, podría ser proferido como una objeción. En (3) con naturalmente el hablante inhibe la posible objeción Pero eso no significa que haya tantos absolutos como seres, lo que sería absurdo. 
La configuración de intersubjetividad sobre la que reposa este uso es bastante compleja. Constitutivo de esta configuración es, primeramente, la capacidad de ponerse en el lugar del otro y de imaginar cómo el otro interpreta mi propio discurso. Estos es, el Yo es capaz de proferir el discurso, interpretarlo desde su propia perspectiva y, al mismo tiempo, desde la perspectiva del Otro, de su interlocutor. En términos más generales: la construcción de sentido en el propio sujeto ya es intersubjetiva. Esta capacidad es indispensable para poder calcular una posible objeción por parte del interlocutor y anticiparse a ella. En segundo lugar, el hablante inhibe la posible objeción profiriendo una aserción, cuyo contenido se presenta, mediante el uso de naturalmente, como algo intersubjetivamente válido: tanto para mí como para ti, como para cualquier otro sería absurdo pensar que haya tantos absolutos como seres.

Ateniéndonos a lo expuesto, el itinerario que seguiremos en las próximas páginas es el siguiente: en la próxima sección presentaremos brevemente los aspectos esenciales de la noción fenomenológica de la intersubjetividad; posteriormente, en la sección 3, propondremos una definición de la intersubjetividad como categoría codificada por la gramática; la sección 4 la dedicaremos a analizar las diferentes configuraciones de intersubjetividad codificadas y convocadas por algunos marcadores de evidencia, así como a explicitar la relación entre estas configuraciones y los efectos argumentativos de los mismos. El trabajo finaliza con una reflexión general sobre la relación entre la intersubjetividad y la argumentatividad.

\section{El CONCEPTO DE LA INTERSUbJetividAd}

Retomemos nuestra reflexión sobre el concepto de la intersubjetividad. Como dijimos, este concepto no es algo nuevo en la lingüística. Relativamente nuevo es, no obstante, el reconocimiento de que está codificado gramaticalmente, es decir, que forma parte del contenido semántico de muchos elementos y construcciones gramaticales (cf. v.gr., Sanders/Sweetser 2009; Sanders/Sanders/Sweetser 2009; Verhagen 2005).

Hemos mencionado, también, que la definición del concepto no es uniforme, sino que oscila, de un modo general, entre dos grandes tendencias (cf. Duranti 2010). Por una parte, se entiende como conocimiento compartido entre dos o más sujetos. En este sentido, la intersubjetividad es 
un resultado de nuestras prácticas comunicativas (o mejor dicho: al cual podemos llegar a través de ellas). Esta lectura del concepto es hoy en día seguramente la dominante en amplios sectores de la lingüística y de otras ciencias humanas y sociales, especialmente en las disciplinas que tienen como objeto de estudio la conversación, el diálogo o el discurso (cf. Duranti 2010, 4-6).

Esta noción de intersubjetividad no es, sin embargo, a nuestro juicio, la más adecuada para caracterizar la gramática, por un motivo bastante obvio: si concebimos la intersubjetividad como el conocimiento compartido que resulta de nuestras prácticas comunicativas actuales, no podemos, al mismo tiempo, considerarla como un prerrequisito para ellas (cf. Duranti 2010, 9). Y la existencia de una gramática - entendida como un sistema simbólico convencional, es decir, ya convencionalizado - lo es. La gramática codifica, para así decirlo, una intersubjetividad ya existente y no una intersubjetividad que todavía tiene que ser construida por nuestras prácticas comunicativas en curso. Por esta razón, optamos en el presente trabajo por una definición fenomenológica del concepto de intersubjetividad, la segunda tendencia a la que hacíamos mención.

En la Fenomenología y, especialmente, en Husserl, ${ }^{3}$ la intersubjetividad es la cualidad esencial de la existencia del ser humano, constitutiva tanto del propio sujeto como de la noción de un mundo objetivo.

Para explicar cómo se constituye la intersubjetividad en el propio sujeto (y no en la comunicación, pues es una condición para su posibilidad), Husserl sitúa en el centro de su reflexión filosófica la esfera primordial del Yo. Esta esfera, caracterizada por la consciencia del propio cuerpo (entendido como Leib, es decir, como cuerpo vivo y vivido por mí), representa la esfera de lo mío propio y todo lo que la traspasa algo extraño al Yo. A partir de ahí, Husserl intenta, en varios pasos, describir cómo se crea, en el sujeto, la referencia a todo lo que es extraño al Yo, al "no-yo" (a lo "no-egóico", "das Ich-Fremde"). En un primer paso, percibo la presencia de otro cuerpo que se parece al mío. Esta semejanza me lleva a una asociación por emparejamiento ("Paarungsassoziation") entre mi cuerpo y el otro cuerpo percibido, lo que a su vez me motiva a atribuirle al cuerpo percibido la cualidad de cuerpo vivo ("Leib"), así como la posesión de una consciencia sobre su cuerpo vivo ("Leibbewußtsein"), a imagen y semejanza de mí mismo. Y, finalmente,

3. Para la exposición del concepto de intersubjetividad en Husserl hemos tomado como texto base Husserl (2002) que corresponde a sus Meditaciones Cartesianas V. 
considero que este proceso de asociación por emparejamiento es recíproco, es decir, atribuyo al otro la facultad de hacer lo mismo al percibir mi cuerpo:

"Leicht verständlich ist auch die Art, wie eine solche Fremdappräsentation im beständigen Fortgang der wirksamen Assoziation immer neue appräsentative Gehalte liefert (...). Den ersten bestimmten Gehalt mu $\beta$ offenbar das Verstehen der Leiblichkeit des Anderen und seines spezifisch leiblichen Gehaltens bilden: das Verstehen der Glieder als tastend oder auch stoßend fungierende Hände, als gehend fungierende Füße, als sehend fungierende Augen usw (...). In weiterer Folge kommt es begreiflicherweise zur Einfüblung von bestimmten Gehalten der böheren psychischen Sphäre. Auch sie indizieren sich leiblich und im außenweltlichen Gehaben der Leiblichkeit, z.B. als äußeres Gehaben des Zornigen, des Fröblichen, etc. - wobl verständlich von meinem eigenen Gehaben her unter ähnlichen Umständen." (Husserl 2002, 198-199). ${ }^{4}$

En síntesis, la intersubjetividad en Husserl hace referencia a la capacidad esencial del sujeto de ponerse en el lugar del otro, de cambiar de lugar („Platzswechseln“). Al hacerlo, extiendo al otro mis características y capacidades. Entre ellas se encuentran todas las competencias psicológicas y cognitivas superiores: el raciocinio lógico, el establecimiento de relaciones causales entre entidades percibidas, etc.

Dicha capacidad constituye una condición para la posibilidad del uso del lenguaje, y esto en un doble sentido. Por un lado, desde una perspectiva evolutiva (tanto filo- como ontogenéticamente), el desarrollo del lenguaje, como un sistema simbólico convencional, presupone el dominio de lo que se denomina un conocimiento de tercer orden, esto es, yo sé que tú sabes que yo sé (cf. Zlatev 2008: 232-237; Itkonen 2008: 288-290). Esto, por su vez, significa reconocer al Otro como agente mental, dotado de las mismas capacidades que yo poseo (incluso de la capacidad de reconocer lo mismo en mí).

4. "Puede también entenderse fácilmente el modo como semejante apresentación de lo otro, en el constante progreso de la asociación activa, va proporcionando siempre nuevos contenidos apresentativos; o sea, cómo trae a conocimiento definido los contenidos cambiantes del otro ego (...). Tiene, evidentemente, que constituir en el primer contenido definido la comprensión de la corporalidad viva del otro y de su comportamiento específicamente corporal: la comprensión de los miembros como manos que actúan palpando o empujando, como pies que actúan andando, como ojos que actúan viendo, etcétera (...). Más adelante se llega, en forma comprensible, a la «endopatía» de contenidos definidos de la «esfera psíquica superior». También éstos dan indicio corporal de sí, y lo hacen en el comportamiento exterior y mundano de la corporalidad, por ejemplo: como conducta exterior de quien está encolerizado, de quien está alegre, etcétera; conducta que se puede comprender bien desde mi propio comportamiento en circunstancias afines". (Edmund Husserl. Meditaciones Cartesianas. Mexico: Fondo de Cultura Economica, 2005. Traduccion de Jose Gaos y Miguel Garcia- Baro: 168-169). 
Por otro lado - y este es el aspecto que más nos interesa en el presente trabajo -, la intersubjetividad es un elemento constitutivo esencial de la base sobre la que erguimos nuestra conceptuación lingüística del mundo. Esto significa que la conceptuación del mundo mediante el lenguaje no se lleva a cabo por un sujeto aislado, nomádico, sino desde la perspectiva de un sujeto trascendente que incluye, como algo esencial en su visión y experiencia del mundo, siempre ya la presencia de la perspectiva del Otro. En resumidas cuentas: el sujeto conceptuador es, en sí mismo, siempre ya un sujeto diádico.

De ello se desprende, necesariamente, que la intersubjetividad forma parte de la estructura semántica del lenguaje, en general, y de la gramática, en particular. Esto no significa, no obstante, que su presencia e importancia sean siempre fácilmente identificables, puesto que, en muchos casos, la intersubjetividad no es nombrada directamente y permanece, más bien, como un elemento implícito de la conceptuación. Es lo que ocurre, por ejemplo, con los conceptos de tiempo y espacio codificados por la gramática. Cuando, al proferir un enunciado como Vi a Juan, ubico temporalmente el evento nombrado con relación al momento presente, el momento presente en cuestión no me pertenece exclusivamente a mí, sino que incluyo, tácitamente, en él también a mi interlocutor. La temporalidad generada, es por tanto, diádica, intersubjetiva y no nomádica, aunque no haya ningún elemento lingüístico que explicite esta cualidad. Lo mismo sucede, para dar otro ejemplo, con el uso de los demostrativos. Cuando utilizo este, no sitúo un objeto en el espacio tan sólo con respecto a mi posición, sino que, al mismo tiempo, incluyo, tácitamente, también la posición del otro. Así, el enunciado Este libro puede ser usado cuando tanto yo como mi interlocutor estamos, al mismo tiempo, cerca del libro o cuando yo estoy cerca y mi interlocutor más alejado y, por el contrario, nunca podrá ser usado cuando sólo mi interlocutor está cerca del libro. En cualquier caso, de un modo u otro, mi interlocutor nunca deja de ser parte constitutiva de la escena. ${ }^{5}$

Existen, por el contrario, muchas otras construcciones gramaticales que evocan la intersubjetividad de una forma más explícita, presentándola, claramente, como una faceta integrante de la estructura conceptual que codifican:

5. Sobre el carácter diádico de los conceptos temporales y espaciales, véase Weinrich (1988: 82-83). 
(4) a. Yo sé muy bien lo que tú piensas al respecto.

b. Se trata de un niño que observa cómo dos viejos vecinos juegan ajedrez en un callejón. El pequeño se obsesiona con la situación y llega a identificarse con uno de los peones al que le falta un pedazo. Tanto así que decide robarlo para protegerlo.

El predicado de pensamiento (sé) y su complemento oracional (que tú piensas) evocan en (4a) explícitamente una determinada configuración de intersubjetividad, en la que el hablante concibe a su interlocutor como sujeto mental que piensa algo respecto de algo. De forma análoga, en (4b), el nexo concesivo Tanto así que y el predicado decide indican que el hablante conceptúa al oyente como un ente capaz de razonar sobre una dada situación y actuar intencionalmente. En ambos casos, las respectivas configuraciones de intersubjetividad constituyen un aspecto focalizado de la semántica de dichas construcciones y elementos gramaticales. Como señala Zlatev (2008: 236), para que enunciados como en (4) tengan sentido, hemos de ser capaces de pensar la intersubjetividad que codifican.

Como ya mencionamos, los marcadores de evidencia pertenecen, en cuanto al grado de explicitación de la intersubjetividad, a este segundo grupo. Antes de proceder al análisis de las diferentes configuraciones de intersubjetividad que evocan, intentaremos, en la próxima sección, consolidar un poco más los fundamentos teóricos que nos permiten considerar la intersubjetividad como una categoría semántica codificada gramaticalmente. Para ello, recurriremos a las nociones de Ground y Grounding, desarrolladas en el seno de la Gramática Cognitiva.

\section{LA INTERSUBJETIVIDAD Y EL ANCLAJE CONCEPTUAL}

Como es sabido, el término 'base conceptual' (Ground) designa, en la Lingüística Cognitiva, el locus de la conceptualización, esto es, el lugar desde el cual experimentamos el mundo y construimos nuestras categorías conceptuales, especialmente aquellas codificadas por el lenguaje. A él pertenecen el evento comunicativo, sus participantes (hablante y oyente), la interacción que se establece entre ellos, así como las circunstancias concretas en las que se realiza el evento (particularmente, su contexto espacio-temporal) (cf. Langacker 1987: 126-128; 2008: 259-264). 
Por 'anclaje' (Grounding) se entiende, por su parte, el proceso epistémico que tiene como efecto la especificación del significado de los elementos lingüísticos que usamos en un enunciado con relación a la base conceptual. Así, por ejemplo, mientras que el sustantivo libro denota un tipo de cosas de las que existen muchas instancias posibles, el grupo nominal este libro designa un ejemplar, es decir, un libro determinado identificado por el hablante y el oyente en una situación particular. Los elementos lingüísticos que desempeñan la función de anclaje reciben el nombre de 'elementos o predicaciones de anclaje'. Los más comunes son los artículos, los demostrativos y los cuantificadores, para el anclaje nominal (cf. v.gr. Langacker 2008: 273-296) y los morfemas de tiempo, modo, persona, etc., en el caso del anclaje verbal (cf. v.gr. Langacker 2008: 296-309).

Especialmente en sus obras más recientes, Langacker reconoce de un modo más explícito que un aspecto constitutivo esencial de la base es su intersubjetividad: ${ }^{6}$

In principle, an expression's conceptualizing meaning always incorporates the conceptualizing presence who apprehends and construes the situation described. ... Minimally, subjectively construed elements include the speaker, and secondary the addressee, who employ the expression and thereby apprehend its meaning. (Langacker 2006: 18).

En la base, tanto el hablante como el oyente actúan siempre como sujetos conceptuadores, en un proceso constante de atención recíproca (Langacker 2008: 262). Toda conceptuación durante un evento comunicativo lleva en cuenta la presencia y la perspectiva del otro. Esto es, no se dirige tan sólo al otro, sino que parte también de la presencia y la perspectiva del otro, como elementos fundamentales de su génesis.

En el presente trabajo defendemos la tesis de que ciertos elementos y construcciones lingüísticas funcionan como predicaciones de anclaje que relacionan el evento comunicativo directamente con la intersubjetividad de la base. Entre ellas se encuentran, como vimos arriba en (4), predicados de pensamiento y complementos oracionales, nexos causales y consecutivos $\mathrm{y}$, en particular también, marcadores de evidencia.

6. Zlatez (2010) subraya el carácter eminentemente fenomenológico de la Gramática Cognitiva de Langacker. 
En su calidad de predicaciones de anclaje, los marcadores de evidencia activan un cierto aspecto constitutivo de la intersubjetividad de la base, y 'anclan' al mismo el contenido del enunciado al que se refieren. En algunos casos, el aspecto activado es la propia percepción inmediata de los interlocutores:

(5) A: - iEl blog ha quedado divino! iLa plantilla es guapísima!

B: - Desde luego. Sobre todo me gusta el detallito del corazón en lugar de la 'b'.

Con el uso de desde luego, el interlocutor B señala que existe intersubjetividad perceptiva con respecto al contenido de los enunciados proferidos por A (función que podemos parafrasear como sigue: 'lo que tú dices que ves, lo veo yo del mismo modo'). Repárese que B prosigue el evento comunicativo con un enunciado que se refiere a un elemento directamente perceptible (el corazón).

En otros casos, en cambio, el aspecto constitutivo de la intersubjetividad de la base, convocado por el marcador, tiene un carácter puramente epistémico. Es lo que ocurre, como vimos arriba, con naturalmente:

(6) Cuando me introducen al calabozo-ventana, me preguntan:

A: - ¿Prefiere usted seguir en esta celda solo, o que lo reunamos con sus compañeros?

B: - Deseo estar con mis compañeros, naturalmente.

A: - Bueno, entonces vamos para allá -me responde el jefe de los guardias.

En (6) (ejemplo repetido de (2)), B ancla su enunciado al ámbito del conocimiento común - o incluso, universal - que se tiene sobre las cosas, indicando, así, que es también un conocimiento compartido por su interlocutor ('yo sé que tú en mi lugar también considerarías natural desear estar con tus compañeros').

Dependiendo del aspecto concreto de la intersubjetividad de la base, activado por un determinado marcador, tendremos diferentes configuraciones de intersubjetividad. De ellas nos ocuparemos de una forma más sistemática en la próxima sección. 


\section{Marcadores de EVIDENCIA y CONFIGURACIONES DE INTERSUBJETIVIDAD}

Básicamente, los marcadores claro, desde luego, naturalmente y por supuesto producen tres tipos distintos de anclaje de intersubjetividad: (i) el anclaje perceptivo, (ii) el anclaje contextual (o discursivo) y, finalmente, (iii) el anclaje epistémico. No existe una correlación estricta entre estos diferentes tipos de anclaje y los marcadores citados, pero sí algunas tendencias notables que vamos ir describiendo a continuación.

Como hemos visto, el anclaje perceptivo se lleva a cabo cuando el hablante incorpora el contenido de un enunciado al ámbito de la percepción común. Es el significado más usual del marcador desde luego. Veamos algunos ejemplos.

(7) A: - En este piso nunca da el sol.

B: - Desde luego, ihace un frío insoportable!

En (7), así como más arriba en (5), el hablante B ancla el contenido del enunciado proferido por su interlocutor (En este piso nunca da el sol) al ámbito de la percepción común. Obsérvese que en los dos casos mencionados el contenido anclado corresponde a un objeto o estado de cosas perceptible o experimentable desde el centro deíctico actual y que, por lo tanto, puede ser observado al mismo tiempo por los dos interlocutores.

En otros casos, por el contrario, desde luego se refiere a algo que solamente ha sido vivenciado por el hablante:

(8) (a) Pese a todo, este invento no es la panacea: el propio fabricante no garantiza que la cama acabe con los ronquidos, al igual que no lo hacen los numerosos métodos hasta abora desarrollados, desde medicamentos hasta el tradicional chasquido de la lengua que acalla por unos instantes los "suspiros guturales". "Pero, desde luego, mejora", aseguró la investigadora del sueño Carina Blomberg.

(b) Sabemos que el proceso de maduración depende, desde luego, de la actividad del cerebro, de la cantidad y calidad de patrones sensoriales (estímulos) y de la educación recibida. ${ }^{7}$

7. Este ejemplo se ha extraído de una entrevista a un especialista en neurociencias, que a través de sus respuestas nos hace partícipes de los conocimientos adquiridos en sus investigaciones. 
En (8a y b), el contenido anclado por desde luego ha sido vivenciado por el respectivo hablante pero no por su interlocutor. Es especialmente en estos casos que podemos apreciar con más nitidez el efecto intersubjetivo causado por el uso de este marcador. El hablante, mediante el uso de desde luego, incorpora al oyente a la esfera de su propia percepción y experimentación: 'si tú estuvieses en mi lugar, habrías percibido y experimentado lo mismo que yo percibí y experimenté'. Con ello se crea, para sí decirlo, a posteriori, una especie de centro deíctico dislocado (o virtual).

El mismo efecto se manifiesta en enunciados reactivos, como se puede apreciar, por ejemplo, en (9):

(9) A: - No se trata de reír, se trata sencillamente de hacer algo que es importante, ¿no?, que es la creación del primer grupo cementero nacional y hacerlo de una manera ordenada, de una manera lógica y de una manera coherente, y creo que es una oportunidad que tenemos por delante, y yo, por todo lo que sea crear un grupo cementero o cualquier otra empresa lógica y ordenadamente y que sea buena para el país, pues voy a tratar de seguir luchando el tiempo que haga falta, soy bastante pesado, sí.

B: - Desde luego. Todo el mundo espera que usted haga algo al respecto de este tema.

En este caso, quien replica se adscribe a sí mismo a la esfera de su interlocutor, corroborando las observaciones que éste último hace y dándole así, en última instancia, razón en sus argumentos y conclusiones (cf. Martín / Portolés 1999: 4152).

El anclaje contextual o discursivo ocurre cuando el hablante, mediante el uso de un marcador, incorpora el contenido de un enunciado al ámbito de un horizonte interpretativo común, relativo al discurso previo: 'reivindico el carácter intersubjetivo de lo que infiero, porque el contexto así me lo impone y, consecuentemente, te lo habrá impuesto a ti también (o me lo habría impuesto a mí si estuviese en tú lugar)'. Es el tipo de intersubjetividad codificado normalmente por el marcador por supuesto.

(10) A: - ¿Has hecho los deberes?

B: - Por supuesto.

Con por supuesto, B convoca un determinado conocimiento contextual, lo presenta como intersubjetivamente válido y establece una relación de consecuencia necesaria entre dicho conocimiento y la respuesta positiva a 
la pregunta absoluta de A. El conocimiento activado actúa, por lo tanto, como una premisa (incuestionada), que conduce a una única conclusión aceptable. En (10), dicho conocimiento puede ser parafraseado de la forma siguiente: 'tú y yo sabemos que siempre hago los deberes'.

Frecuentemente, por supuesto es utilizado para bloquear, de antemano, una posible (o, incluso, previsible) inferencia del interlocutor:

(11) Seguramente los caraquistas diremos pesadeces jugando con los turcos y las hallacas, pero en realidad la eliminación del Magallanes tan temprano es la peor de todas. No es, por supuesto, para pedir que nadie, sobre todo nadie, intervenga al equipo, pero estoy de acuerdo con el sensato Wilmer Zoteranis, al menos el doctor Latouche debería pensar en renunciar.

Las críticas expresadas por el hablante nos podrían hacer pensar (y decir) que está sugiriendo que alguien intervenga al equipo. Esta posible inferencia, no obstante, es bloqueada, anticipadamente, mediante la afirmación de lo opuesto. Obsérvese que la sustitución de una posible e incluso previsible inferencia por su opuesto tan sólo puede contar con perspectivas de éxito si dicho opuesto es ratificado como algo válido en el contexto del evento comunicativo actual. Esta es, precisamente, la función de por supuesto. Con el uso de este marcador, B convoca un determinado conocimiento contextual (que podríamos parafrasear de la siguiente manera: 'tú y yo sabemos que no soy partidario de intervenciones'), lo presenta como intersubjetivamente válido y ancla al mismo en contenido del enunciado al que se remite el marcador (No es, por supuesto, para pedir que nadie, sobre todo nadie...). Como arriba en (10), el conocimiento activado actúa, aquí también, como una premisa (incuestionada), que conduce a una única conclusión aceptable. ${ }^{8}$

La posibilidad de intervenir, mediante el uso de por supuesto, en el proceso inferencial realizado (real- o supuestamente) por el otro manifiesta claramente el valor intersubjetivo de dicho marcador y ratifica, al mismo tiempo, la pertinencia de la definición de la intersubjetividad que hemos ofrecido en la sección precedente, puesto que esta posibilidad implica que

8. En el probable proceso de inferencia 'Premisa 1: Quien critica exige cambios. Premisa 2: el hablante critica el equipo. Conclusión: el hablante exige cambios en el equipo', el uso de por supuesto desactiva la validez de la primera premisa y, consecuentemente, altera el proceso conclusivo. 
el hablante, poniéndose en el lugar del otro, incluye en su intervención discursiva lo que piensa que está en la mente de este último.

El término anclaje epistémico lo reservamos para aquellos casos en los que el contenido de la base que sirve de ancla posee un carácter general, o incluso universal, trascendiendo, así, los límites del contexto comunicativo actual. El marcador que, de una forma más clara, realiza este tipo de anclaje es naturalmente. Veamos algunos ejemplos.

(12) Sorprende también que se infravalore la memoria bistórica, tantas veces conservada en el ámbito familiar, o no se tenga en cuenta el cúmulo de influencias que cada individuo va recibiendo a lo largo de su proceso educativo, en sus lecturas, en sus viajes, en sus distintas experiencias como trabajador o empresario. Y ipor qué no decirlo?, aunque hoy suene a trasnochado, su pertenencia a una determinada clase social. Que no son idénticas a las que observó Marx, naturalmente, afortunadamente, al menos en buena parte de la Europa a la que pertenecemos, pero que siguen siendo reconocibles y que afloran a la superficie-gravemente-cuando nos enfrentamos al problema de la inmigración.

Con el uso de naturalmente, se invoca la existencia de un consenso amplio, general, en torno a la aserción de que las clases sociales no son idénticas a las que observó Marx, esto es, un consenso que trasciende la esfera estricta de una intersubjetividad compartida exclusivamente entre el hablante y el oyente. El conocimiento de la base activado por el marcador puede ser caracterizado a través de las siguientes paráfrasis: como se sabe, como es ampliamente sabido, como es natural, etc. (las clases sociales de hoy no son idénticas a las que observó Marx en su día).

Como se ha descrito para por supuesto, uno de los efectos de sentido más frecuentes de naturalmente en el plano argumentativo es el de actuar como inhibidor de una posible o previsible objeción o inferencia indeseada. En (12), el hablante prevé que su interlocutor podría objetar que hoy en día no existen más clases sociales o que no existen más en su sentido histórico, etc. Como hemos dicho arriba y ratificamos aquí, la intersubjetividad, en el sentido expuesto en la sección 3, constituye la condición para la posibilidad de salir al paso de una posible objeción por parte del interlocutor: el hablante es capaz de ponerse en el lugar de su (real o potencial) interlocutor e incluir en su razonamiento lo que está o pudiera estar en la mente del otro.

Nos resta analizar, para concluir esta sección, la intersubjetividad evocada por el marcador claro. Al contrario de lo que hemos constatado 
con respecto a desde luego, por supuesto y naturalmente, claro no puede ser adscrito a un único tipo específico de configuración de intersubjetividad. En ello radica, sin duda, una de las causas de su mayor frecuencia de uso en comparación con los otros tres marcadores, especialmente en textos orales (Martín / Portolés 1999: 4155-4156).

No resulta difícil, así pues, encontrar ejemplos de las tres configuraciones de intersubjetividad que venimos analizando:

(13) Proponemos también la modificación de los artículos transitorios de la minuta para disponer que la Entidad inicie funciones el primero de enero del año dos mil, no el dos mil uno, como inicialmente se proponía. Pero, claro, que la revisión de los ejercicios de noventa y ocho, noventa y nueve y dos mil se haga conforme a las actuales reglas vigentes para permitir un periodo de transición y de aprendizaje conforme a las nuevas normas y procedimientos.

En (13), el contenido activado por claro puede ser parafraseado como sigue: 'tú y yo sabemos que es normal que la revisión de los ejercicios se ha de hacer según las actuales reglas'. Este contenido de la base no puede ser considerado como algo perteneciente al ámbito del conocimiento general ni tampoco representa, obviamente, un aspecto de la percepción común del hablante y del oyente. Se trata, más bien, de un contenido intersubjetivamente válido en el contexto específico del evento comunicativo (legislación de ciertas entidades).

Con claro podemos evocar también contenidos que pueden ser adscritos al ámbito del conocimiento general o universal:

(14) Dice Cecilia Garzón: — "Licenciada Margarita Zavala, ¿qué se necesita para pertenecer al Instituto de la Mujer?", y pone entre paréntesis: "aparte de ser mujer, claro".

Finalmente, tampoco son raros los casos en los que se utiliza claro para realizar un anclaje perceptivo:

(15) - ¿La luz era del triángulo o un reflejo de la luna?

- No. Era del triángulo. Estoy completamente segura que era de él porque eso no era ningún reflejo ni nada, y era completamente blanco y luminoso. Y entonces en un segundo desapareció. Vi la dirección que tomaba pero ya nada más.

- ¿Pero desapareció, se oculto a la vista, había nubes o desapareció, se fue?

- Se fue. Se fue en otra dirección.

- ¿A que velocidad? 
- Muy rapidísimo; un segundo o menos de un segundo, rapidísimo.

- ¿Y aumento el ruido al irse?

- No, al irse disminuyó. Era un ruido constante, pero al irse, claro, disminuyó.

Como se puede apreciar, el contenido anclado mediante el uso del marcador describe, en este caso, un estado de cosas directamente perceptible por el hablante (Era un ruido constante, pero al irse, disminuyó). Al igual que se ha descrito para desde luego, el hablante, mediante el uso de claro, incorpora al oyente a la esfera de su propia percepción y experimentación: 'si tú hubieses estado presente, habrías percibido lo mismo que yo". Esta validez intersubjetiva de lo percibido se adquiere anclando el estado de cosas particular a una experiencia y un conocimiento generales sobre la percepción de ruidos: 'cuando el objeto que produce el ruido se aleja, el ruido disminuye'.

El análisis que hemos desarrollado en esta sección nos permite concluir que los marcadores de evidencia desde luego, naturalmente, por supuesto y claro actúan claramente como elementos o predicaciones de anclaje. Su función concreta reside en anclar el contenido del enunciado al que remiten a un aspecto constitutivo de la intersubjetividad de la base de conceptuación. Si los comparamos a las predicaciones de anclaje nominal o verbal, tradicionalmente estudiados en la literatura, se nos revelan algunas diferencias importantes que constituyen la especificidad del anclaje producido por estos marcadores.

Así, por una parte, mientras que el anclaje nominal y el verbal tienen como efecto una especificación del significado de elementos lingüísticos que componen la oración (el nombre y el verbo, respectivamente), el anclaje producido por los marcadores abarca siempre el significado total de un enunciado o, como veremos con más atención en la próxima sección, incluso el significado de varios enunciados a la vez. Se trata, pues, de un anclaje que podríamos clasificar como textual.

Una segunda diferencia importante, reside, por otra parte, en el elemento de la base que en cada caso es activado para actuar de ancla. A este respecto, hemos de constatar que, aunque la base sea, como dijimos arriba, inherentemente intersubjetiva, diádica, no todas las predicaciones de anclaje convocan explícitamente esta cualidad. En muchos casos la referencia es más implícita que explícita, como ocurre, por ejemplo, con la categoría 
verbal del tiempo o con los demostrativos, elementos a los que nos hemos referido brevemente en la sección anterior. Los marcadores analizados, en cambio, convocan directa e explícitamente algún aspecto constitutivo del carácter intersubjetivo de la base de conceptuación. Dependiendo de la naturaleza concreta de dichos aspectos, hemos identificado tres tipos de anclaje de intersubjetividad: perceptivo, contextual y epistémico.

\section{LA INTERSUBJETIVIDAD Y LA ARGUMENTATIVIDAD}

En esta sección nos ocuparemos de la relación entre la intersubjetividad y la argumentatividad en su calidad de aspectos constitutivos de la semántica de los marcadores de evidencia. A este respecto, argüimos en la introducción al presente trabajo que los efectos de sentido producidos por los marcadores de evidencia reposan sobre las configuraciones de intersubjetividad generadas mediante el uso de los mismos. Para concretar un poco más esta tesis se hace necesario presentar brevemente algunos conceptos fundamentales de la Teoría de la Argumentación (Anscombre / Ducrot 1986; Portolés 2008).

En su versión más actual, que Anscombre y Ducrot (1986: 87-94) denominan "argumentatividad radical", la Teoría de la Argumentación postula que todas las frases (y, de un modo general, todas las unidades lingüísticas) están dotadas, de una forma u otra, de una significación argumentativa. Esto se manifiesta, especialmente, en el hecho de que las frases favorecen una serie de continuaciones del discurso, mientras que dificultan otras. Para explanar este fenómeno, Portolés (2008: 78), analiza los siguientes enunciados:

(16) a. Este chico está gordo. Quiere adelgazar.

b. Esta casa es nueva. Está recién pintada.

c. Está lloviendo. Llévate el paraguas.

Portolés señala que, por lo general, las personas gruesas quieren adelgazar, las casas nuevas acostumbran a estar recién pintadas y cuando llueve es normal que uno se cubra con un paraguas. De ello podemos concluir, según este autor, que los segundos enunciados mantienen la orientación argumentativa de los primeros. 
Si el chico gordo, por el contrario y contra lo esperado, no quiere perder peso, podremos decir lo siguiente:

(17) Este chico está gordo. Sin embargo, no quiere adelgazar.

Según Portolés (2008: 78), el marcador sin embargo relaciona el primer enunciado con el segundo e indica por su significación, que contrariamente a lo que se debería esperar de la orientación argumentativa de este chicho está gordo, el muchacho no desea adelgazar. El marcador posee, por tanto, un efecto antiorientativo.

Ahora bien, no siempre el marcador sin embargo provoca una ruptura de la orientación argumentativa del enunciado precedente:

(18) Alicia es madrileña. Sin embargo, no le gusta el metro.

En este caso - señala Portolés (2008: 79) - la significación de Alicia es madrileña no está orientada ni hacia un placer o desplacer por usar el metro. Con ello, resta considerar que es la inclusión del marcador lo que indica que existe una antiorientación argumentativa de Alicia es madrileña con respecto a 'gustar el metro'. De este análisis, Portolés (2008: 79) concluye que los marcadores "proporcionan por su significación una serie de instrucciones para construir el sentido de los enunciados en los que se encuentran”.

Indaguemos un poco más en qué radica este sentido construido por los marcadores. Para que se pueda establecer una antiorientación argumentativa entre 'gustar el metro' y Alicia es madrileña, es imprescindible que, en el momento de la enunciación, se active la información 'a los madrileños mayoritariamente les gusta el metro’. Además, es necesario que dicha información pertenezca al ámbito del conocimiento común, esto es, que tenga validez intersubjetiva. Este conocimiento general, intersubjetivamente válido, no forma parte de la significación de ninguno de los dos enunciados, sino que es activado directamente por el marcador sin embargo.

Lo mismo puede ser afirmado con respecto los enunciados en (17). Pues, aunque, como bien apunta Portolés, podamos considerar que la significación del propio enunciado Este chico está gordo favorece la activación de la información de que 'los gordos generalmente quieren adelgazar', será la inclusión del marcador sin embargo lo que active directamente esta 
información y ratifique su aplicabilidad a la construcción de la relación argumentativa en este caso particular. Esta información general, activada por el marcador, corresponde a lo que hemos denominado ancla en la sección precedente.

Volvamos ahora, aparejados ya con algunos conceptos de la Teoría de la Argumentación, al análisis de nuestros marcadores de evidencia.

Como hemos visto en la sección precedente, uno de los efectos argumentativos más frecuentes de los marcadores de evidencia es el de actuar como inhibidores de una posible o previsible objeción por parte del interlocutor. Observemos, nuevamente, el enunciado de (12), que para facilitar su análisis, reproducimos a continuación:

(19) Sorprende también que se infravalore la memoria histórica, tantas veces conservada en el ámbito familiar, o no se tenga en cuenta el cúmulo de influencias que cada individuo va recibiendo a lo largo de su proceso educativo, en sus lecturas, en sus viajes, en sus distintas experiencias como trabajador o empresario. Y ¿por qué no decirlo?, aunque boy suene a trasnochado, su pertenencia a una determinada clase social. Que no son idénticas a las que observó Marx, naturalmente, afortunadamente, al menos en buena parte de la Europa a la que pertenecemos, pero que siguen siendo reconocibles y que afloran a la superficie-gravemente-cuando nos enfrentamos al problema de la inmigración.

El referido efecto argumentativo, surge, especialmente, cuando el enunciado afectado por el marcador se encuentra orientado contraargumentativamente en relación con el que le precede (Martín / Portolés 1999: 4149-4158). En el caso aquí analizado, la pertenencia a una determinada clase social no favorece la afirmación de que ya no existen las clases sociales postuladas por Marx. ¿Cuál es el aporte semántico de naturalmente al enunciado en el que se utiliza? En primer lugar, como vimos, el marcador transforma lo que podría ser una opinión meramente individual en una afirmación dotada de una validez amplia, general: 'yo sé que tú sabes que yo sé (y, en general, todo el mundo sabe) que las clases hoy en día no son las mismas que observó Marx'. En segundo lugar, el anclaje intersubjetivo, producido mediante el uso de naturalmente, le permite al hablante utilizar esta afirmación, en el plano argumentativo, como una contraargumentación general, esto es, adscrita a la esfera de todos los interlocutores. Esto torna desnecesaria - y por lo tanto, inhibe - la enunciación de una posible objeción por parte del oyente. En síntesis: el efecto argumentativo de la inhibición de una posible objeción presupone el anclaje intersubjetivo del enunciado con el que se pretende producir tal efecto. 
El anclaje intersubjetivo es imprescindible también para llevar a cabo la inhibición de inferencias no deseadas:

(20) Seguramente los caraquistas diremos pesadeces jugando con los turcos y las hallacas, pero en realidad la eliminación del Magallanes tan temprano es la peor de todas. No es, por supuesto, para pedir que nadie, sobre todo nadie, intervenga al equipo, pero estoy de acuerdo con el sensato Wilmer Zoteranis, al menos el doctor Latouche debería pensar en renunciar.

En (20) (ejemplo repetido de 11), el hablante, por medio del uso de por supuesto, bloquea la posible inferencia de una afirmación que conservaría la orientación argumentativa del segmento anterior del discurso y profiere, en su lugar, un enunciado contraargumentativo. Como mencionamos arriba, este efecto argumentativo tan sólo es posible porque el marcador sustituye, para así decirlo, un tipo de intersubjetividad por otro: un conocimiento compartido de carácter más amplio ('cuando alguien critica a alguien sugiere que se intervenga, se sustituya, etc. al criticado) es remplazado por un consenso más limitado, válido en el contexto del evento comunicativo actual ('tú y yo sabemos que no soy partidario de intervenciones').

El último uso de marcadores de evidencia que queremos analizar para verificar nuestra tesis es el refuerzo de la cooperación comunicativa y, derivada de ella, la construcción de una cortesía positiva entre los interlocutores (Martín / Portolés 1999: 4156). Muy frecuentemente, el hablante consigue este efecto mediante el empleo de desde luego y, especialmente, claro, como se percibe en los enunciados que siguen:

(21) A: - Porque yo me encontraba muy mal. Así como baja. Me encontraba muy mal. Pero cuando vas en pandilla, como no quieres perjudicar a los otros...

B: Claro.

A: Y entonces, yo fui, con dolor de cabeza y todo.

(22) A: - En este piso nunca da el sol.

B: - Desde luego, ihace un frío insoportable!

Es importante observar que el refuerzo de la cooperación y, consecuentemente, de la cortesía positiva, constatable en intercambios como los que ofrecemos en (21) y (22), (este último reproducido de 7), deriva del anclaje intersubjetivo producido por el uso de los marcadores. Mediante su empleo, el hablante confirma la validez de lo dicho anteriormente 
por su interlocutor, adscribe el contenido del enunciado previamente proferido al horizonte de una realidad compartida, contribuyendo, con ello, a la formación de una identificación recíproca, de la percepción de una pertenencia a una misma visión de mundo, a la intensificación de un sentimiento de solidaridad, etc.

\section{Conclusión}

Si el análisis que hemos desarrollado a lo largo de este trabajo es correcto, hemos de concluir que la semántica de los marcadores de evidencia consiste, ante todo, en una serie de instrucciones para especificar el sentido del enunciado en el que se utilizan con respecto a un elemento esencial de la base de conceptualización: la intersubjetividad. Los efectos de sentido que estos elementos provocan en la conexión entre enunciados derivan de esta función especificadora. En síntesis: el anclaje precede a (y posibilita) la función argumentativa.

La intersubjetividad es un elemento constitutivo de la base de conceptuación. Quizás, incluso, sin temor a exagerar, podríamos afirmar que se trata del elemento que determina su esencia. Toda conceptuación que se realiza durante el evento comunicativo es intersubjetiva, diádica, y nunca monádica, en el sentido de que el hablante, al conceptuar el mundo, lo hace siempre e inevitablemente desde una doble perspectiva: desde la propia y desde la que él mismo tendría si ocupase el lugar de su interlocutor. El anclaje intersubjetivo se produce cuando la semántica de un elemento lingüístico hace referencia a esta condición esencial de la base. Como hemos visto, esta es la función principal de los marcadores de evidencia, así como de ciertas construcciones gramaticales (oraciones complejas sustantivas, causales, consecutivas, etc.). El análisis detallado de estos elementos y de las configuraciones específicas de intersubjetividad que codifican va ocupar, sin lugar a dudas, un gran espacio en la investigación lingüística de los próximos años.

Recebido em dezembro de 2012 Aprovado em julho de 2013

E-mails: huelva@unb.br; enriquehuelva@gmail.com 


\section{REFERENCIAS BIBLIOGRÁFICAS}

Anscombre, Jean-Claude \& Ducrot, Oswald. 1986. Argumentativité et informativité. En: Michel Meyer (ed.) De la métaphysique à la rbétorique, essais à la mémoire de Chaïm Perelman avec un inédit sur la logique. Bruxelles : Editions de l'Université de Bruxelles: 79-94.

Duranti, Alessandro. 2010. Husserl, Intersubjectivity and Anthropology. Anthropological Theory, 10, 1: 1-20.

Fuentes Rodríguez, Catalina. 1993a. Claro: modalización y conexión. En: Pedro Carbonero y Catalina Fuentes (eds.) Sociolingüística Andaluza 9. Estudios sobre el enunciado. Sevilla: Universidad de Sevilla: 99-126.

Fuentes Rodríguez, Catalina. 1993b. Desde luego, Por supuesto, Naturalmente. En: Pedro Carbonero \& Catalina Fuentes (eds.) Sociolingüística Andaluza 9. Estudios sobre el enunciado. Sevilla: Universidad de Sevilla: 127-160.

Huelva Unternbäumen, Enrique. En prensa. Construcciones causales con la preposición ante en la lengua española. Zeitschrift für romanische Philologie 129, 3.

Husserl, Edmund. 2002. Konstitution der Intersubjektivität. En: Klaus Held (ed.) Phänomenologie der Lebenswelt. Ausgewählte Texte II. Stuttgart: Reclam: 166-219. [Traducido al español en: Edmund Husserl. Meditaciones Cartesianas. Mexico: Fondo de Cultura Economica, 2005. Traduccion de Jose Gaos y Miguel Garcia-Baro].

ITKONEN, Esa. 2008. The central role of normativity in language and linguistics. En: Jordan Zlatev, Timothy P. Racine, Chris Sinha \& Esa Itkonen (eds.) The Shared Mind. Perspectives on Intersubjectivity. Amsterdam \& Philadelphia: John Benjamins: 279-305.

LANGACKER, Ronald. 1987. Foundations of Cognitive Grammar, vol. 1: Theoretical Prerequisites. Stanford: Stanford University Press.

LANGACKer, Ronald. 2008. Cognitive Grammar. A Basic Introduction. Oxford: Oxford University Press.

Martín Zorraquino, María A. \& Portolés Lázaro, José. 1999. Los marcadores del discurso. En: Ignacio Bosque \& Violeta Demonte (eds.) Gramática descriptiva de la lengua española, Madrid: Espasa: 4051-4213.

Portolés, José. 2008. La teoría de la argumentación en la lengua y los marcadores del discurso. En: María A. Martín Zorraquino \& Estrella Montolío Durán (eds.) Los marcadores el discurso. Madrid: Arco Libros: 71-91. 
Sanders, Ted y Sweetser, Eve. 2009. Introduction: Causality in language and cognition - what causal connectives and causal verbs reveal about the way we think En: Ted Sanders \& Eve Sweetser (eds.) Causal Categories in Discourse and Cognition. Berlin \& New York: Mouton de Gruyter: 1-18.

Sanders, Ted, Sanders, José y Sweetser, Eve. 2009. Causality, cognition and communication: A mental space analysis of subjectivity in causal connectives. En: Ted Sanders \& Eve Sweetser (eds.) Causal Categories in Discourse and Cognition. Berlin \& New York: Mouton de Gruyter: 20-59. Schegloff, Emanuel A. 2006. Interaction: The Infrastructure for Social Institutions, the Natural Ecological Niche for Language, and the Arena in which Culture is Enacted. En: N.J. Enfield \& S.C. Levinson (eds.) Roots of Human Sociality: Culture, Cognition and Interaction. Oxford \& New York: Berg: 70-96.

Verhagen, Arie. 2005. Constructions of Intersubjectivity. Oxford: Oxford University Press.

Verhagen, Arie. 2008. Intersubjectivity and the architecture of the language system. En: Jordan Zlatev, Timothy P. Racine, Chris Sinha \& Esa Itkonen (eds.) The Shared Mind. Perspectives on Intersubjectivity. Amsterdam y Philadelphia: John Benjamins: 307-331.

WeINRICH, Harald. 1988. Textgrammatik der französischen Sprache. Stuttgart: Klett.

Zlatev, Jordan. 2008. The co-evolution of intersubjectivity and bodily mimesis. En: Jordan Zlatev, Timothy P. Racine, Chris Sinha \& Esa Itkonen (eds.) The Shared Mind. Perspectives on Intersubjectivity. Amsterdam \& Philadelphia: John Benjamins: 215-244.

Zlatev, Jordan. 2010. Phenomenology and Cognitive Linguistics. En: Shaun Gallagher \& Daniel Schmicking (eds.) Handbook on Phenomenology and Cognitive Sciences. Dordrecht: Springer: 415-446. 